\title{
Gene expression profile in rat pancreatic islet and RINm5F cells
}

\author{
H Wang1, Y Horikawa ${ }^{1,2,3}$, L Jin ${ }^{1}$, T Narita1, S Yamada1, N Shihara ${ }^{1,2}$, K Tatemoto $^{4}$, \\ M Muramatsu ${ }^{3}$, T Mune ${ }^{3}$ and $\mathrm{J}$ Takeda $^{1,2,3}$ \\ ${ }^{1}$ Laboratory of Molecular Genetics, Department of Cell Biology, Institute for Molecular and Cellular Regulation, Gunma University, Gunma, Japan \\ ${ }^{2}$ Core Research for Evolutional Science and Technology (CREST), Japan Science and Technology Corporation (JST), Kawaguchi, Japan \\ ${ }^{3}$ Department of Diabetes and Endocrinology, Division of Molecule and Structure, Gifu University School of Medicine, Gifu, Japan \\ ${ }^{4}$ Laboratory of Peptide and Protein Research, Department of Molecular Physiology, Institute for Molecular and Cellular Regulation, Gunma University, Gunma, Japan
}

(Requests for offprints should be addressed to Yukio Horikawa, Department of Diabetes and Endocrinology, Gifu University School of Medicine, 1-1 Yanagido, Gifu-city, Gifu 500-1194, Japan; Email: yhorikaw@cc.gifu-u.ac.jp)

\begin{abstract}
To clarify tissue-specificity of pancreatic $\beta$ cells, comparison of mRNA expression in various conditions of the tissue of multiple organisms is important. Although the developed methodologies for mRNA monitoring such as microarray, rely on the growth of dbEST (database of expressed sequence tag), a large number of unknown genes in the genome, especially in the rat, have not been shown to be expressed. In this study, we have established the first database of ESTs from rat pancreatic islet and RINm5F cells. Two cDNA libraries were constructed using mRNAs from rat pancreatic islet and RINm5F cells to cover a wider spectrum of expressed genes. Over 40000 clones were randomly selected from the two libraries and partially sequenced. The sequences obtained were subjected to BLAST database analyses. This large-scale sequencing generated $407103^{\prime}$-ESTs. Clustering analysis and homology search of nucleotide and peptide databases using both $3^{\prime}$ - and 5'-ESTs revealed 10406 non-redundant transcripts representing 4078 known genes or homologs and 6328 unknown genes. To confirm actual expression, the unknown sequences were further subjected to dbEST search, resulting in the identification of 5432 significant matches to those from other sources. Interestingly, of the remaining sequences showing no match, 779 were found to be encoded by exon-intron organization in the corresponding genomic sequences, suggesting that these are newly found as actually expressed in this study. Since many genes are up- or down-regulated in differing conditions, applications of the expression profile should facilitate identification of the genes involved in cell-specific functions in normal and disease states.
\end{abstract}

Journal of Molecular Endocrinology (2005) 35, 1-12

\section{Introduction}

Pancreatic islets play the critical role in the regulation of blood glucose by secreting hormones from endocrine cells that differentiate from common progenitor cells during fetal development (postnatal origin of $\beta$-cell replenishment remains controversial) (Edlund 2002, Bonner-Weir \& Sharma 2002, Dor et al. 2004). Functional defects of $\beta$-cells lead to the development of diabetes mellitus. Since a number of genes are involved in the pathogenesis of impaired insulin secretion, it is important to characterize the expression profile of a set of genes that endow $\beta$-cells with the tissue-specific functions of insulin synthesis and secretion.

Recent genome projects demonstrated a similar number of 30 000-40 000 genes in human and mouse chromosomes, including $\sim 27000$ protein-encoding transcripts for which there was strong corroborating evidence and $\sim 10000$ computationally derived genes with weak supporting evidence (International Human
Genome Sequencing Consortium 2001, Venter et al. 2001, Mouse Genome Sequencing Consortium 2002). Comparison with the transcriptome revealed almost all of the human genes known to be expressed to have orthologues in the mouse genome. The other putatively novel genes in the genome were detected using computer algorithms for transcript prediction. To estimate the accuracy of the power of new gene detection, the results of the gene annotation done by the two human genome efforts were previously compared (Hogenesch et al. 2001). Surprisingly, although a similar number of the total genes was demonstrated, there is little agreement regarding the new genes predicted by the two projects, suggesting that a significant fraction of tissue-restricted transcripts for novel genes remain undiscovered, possibly due to limitations in the computer prediction methods.

As expression analysis of the genes in multiple organisms becomes a major focus in the new era of biology, functional genomics will rely largely on the vast 
sources of subsets of partial cDNA sequences from various tissues that have proven enormously valuable and are deposited as expressed sequence tags (ESTs) in the public databases. The Endocrine Pancreas Consortium has recently constructed human and mouse cDNA libraries from various conditions of endocrine pancreas and generated over 100000 ESTs (Bernal-Mizrachi et al. 2003). We also have collected 20000 ESTs from human normal pancreatic islets and islet tumors, resulting in the identification of $\sim 3000$ new genes expressed in the islets (Takeda et al. 1993, Jin et al. 2003). Such systematic sequencing efforts complement each other and should improve the various methodologies including DNA microarray technology (Scearce et al. 2002) for monitoring differential gene expression in normal and disease states. In addition, the laboratory rat is an indispensable model organism of human diseases, providing a useful tool in experimental medicine and drug discovery. As various spontaneous diabetic rats such as the GK and OLETF rats and the experimental streptozotocin-induced diabetic rat are widely used in pancreatic islet studies, it is important to establish an additional source of rat expressed sequences. However, although 26 millions of ESTs have so far been deposited in the database (dbEST release 031105), approximately $40 \%$ of which are derived from human and mouse, only $2 \cdot 6 \%$ of the sequences are from rat, and none are from pancreatic islets except for the present deposition. In this study, toward elucidation of the entire transcriptome in rat pancreatic islets, we have made two cDNA libraries, one from rat normal pancreatic islets and the other from RINm5F tumor cells having undergone less differentiation (Gazdar et al. 1980, Philippe et al. 1987), and performed a large-scale collection of ESTs. Since a number of the genes are up- or down-regulated in different conditions, a collection of ESTs from these distinct cDNA sources should more effectively cover a wider spectrum of expressed genes, generating a larger pool of nonredundant sequences. In addition, since the insulin content of the less-differentiated RINm5F cells used in this study was previously found to be much lower than that of normal islets (Kayo et al. 1997), direct comparison of the expression profiles of the two cDNA libraries should facilitate the identification of the genes involved in insulin synthesis and secretion as well as in $\beta$-cell differentiation and tumorigenesis.

\section{Materials and methods}

\section{Preparation of rat pancreatic islets}

Pancreatic islets were prepared from male Wister rats by a collagenase digestion method as described previously (Ma et al. 1996). Briefly, under pentobarbital anesthesia, the pancreas was distended by an injection of $10 \mathrm{ml}$
Hank's solution containing $0.3 \mathrm{mg} / \mathrm{ml}$ collagenase (type XI, Sigma-Aldrich, StLouis, MO, USA). Islets were separated by the Ficoll (Amersham, Piscataway, NJ, USA) density gradient method with four layers (27\%, $23 \%, 20.5 \%$, and $11 \%$ of Ficoll dissolved in Hank's solution). After centrifugation at $450 \boldsymbol{g}$ for $15 \mathrm{~min}$, pancreatic islets were concentrated at the interface between the $11 \%$ and $20.5 \%$ Ficoll layers. Islets were then harvested by a pick-up method under a stereomicroscope. Purity of the islets was estimated to be $\sim 99 \%$ by counting the cells immunoreactive to insulin and glucagon antibodies after trypsin treatment of a fraction of the islets collected. The high purity also could be estimated by the frequency of cDNA for major exocrine molecules, such as $\alpha$-amylase, in the entire islet ESTs identified, as described below.

\section{Large-scale cDNA sequencing}

Two unidirectional cDNA libraries were constructed in the Uni-ZAP XR vector (Stratagene, La Jolla, CA, USA) using mRNAs from rat normal pancreatic islets and RINm5F cells. A large set of plasmid DNAs for sequencing was prepared as described (Takeda et al. 1993, Jin et al. 2003). Briefly, the cDNA libraries were excised en masse from the $\lambda$ phage into phagemid particles using the ExAssist phage system (Stratagene), and subsequently transfected into $E$. coli SOLR (Stratagene) for conversion to plasmid forms. Plasmid DNAs were extracted from $E$. coli colonies randomly selected from LB-Amp plates using the Biomek 2000 mini-prep system (Beckman, Fullerton, CA, USA). Single-pass DNA sequencing from the 3 '-end of the inserts was performed using a BigDye Terminator Cycle Sequencing FS Ready Reaction Kit and DNA Sequencer model 3700 (Applied Biosystems, Foster City, CA, USA). Vector sequences were removed from the results using Assembly LIGN software (Oxford Molecular Group PLC, Oxford, UK). Quality assessment of the sequences obtained was performed using PE Sequencing Analysis 3.3 software (Applied Biosystems).

\section{Database analysis of rat pancreatic islet and RINm5F ESTs}

We compared a total of $\sim 40000$ sequences from rat pancreatic islet and RINm5F cells with non-redundant nucleotide and peptide sequences extracted in silico from databases at the National Center for Biotechnology Information (NCBI). Before comparisons, interspersed repetitive sequences such as LINEs were unmasked and removed from the pool using software RepeatMasker (http://ftp.genome.washington.edu/RM/RepeatMasker. html). To assemble sequences sharing a stretch of nucleotide identity, the LaboServer system (World fusion, Tokyo, Japan) was applied to make contigs. 
Representative sequences from each contig then were subjected to BLASTN analysis for sequence homology at nucleotide level against a merged database by the Kiroku program (World fusion). If a query sequence shared over 95\% nucleotide identity and showed a score of more than 400 with any sequences in the database, they were grouped together. The clones without significant match to known sequences in the nucleotide database were re-sequenced from the other end to compare the sequences with those in the peptide database at NCBI using BLASTX program (Altschul et al. 1997), which conceptually translates the query sequence in all six reading frames for comparison. The ESTs identical or highly similar to functionally annotated genes were first classified into seven major categories according to the general functions of the proteins encoded, and then further classified into subcategories according to their specific functions.

\section{Semi-quantitative RNA expression analysis}

To ascertain the level of mRNA expression of the ESTs from rat normal islets and RINm-5F cells, real-time quantitative reverse transcriptase (RT)-PCR was carried out using an ABI PRISM 7900HT Sequence Detection System (Applied Biosystem). Total RNA was extracted from pooled islets isolated from normal rats and RINm5F cells, using an RNeasy Mini Preparation Kit (Qiagen, Valencia, CA, USA) according to the manufacturer's instructions. TaqMan primers and probes were designed using Primer Express software purchased from Applied Biosystems. TaqMan reactions were performed in a reaction volume of $20 \mu \mathrm{l}$ using components supplied in a TaqMan PCR reagent kit. Each reaction consisted of $10 \mu \mathrm{l}$ TaqMan Universal Master Mix, $900 \mathrm{nM}$ of each amplification primer, and $250 \mathrm{nM}$ corresponding TaqMan probe. Each sample was run for an initial $2 \mathrm{~min}$ at $50^{\circ} \mathrm{C}$ and $10 \mathrm{~min}$ at $95^{\circ} \mathrm{C}$, followed by 40 cycles at $95^{\circ} \mathrm{C}$ for $15 \mathrm{~s}$ and at $60{ }^{\circ} \mathrm{C}$ for $1 \mathrm{~min}$. Amplification data were collected by the 7900HT Sequence Detector and analyzed using Sequence Detection System software. The RNA concentration was determined from the threshold cycle at which fluorescence is first detected, cycle number being inversely related to RNA concentration.

\section{In situ hybridization}

ESTs showing marked differences in frequency between islet and RINm5F cells were subjected to analysis of mRNA distribution in the pancreas by in situ hybridization. Paraffin embedded blocks and sections of normal rat pancreas for in situ hybridization (ISH) were obtained from GENOSTAFF, Inc. (Tokyo, Japan). The pancreases of male Wistar rats 8 weeks old (CREA Tokyo, Japan, Inc.) were dissected after perfusion, fixed by Tissue Fixative (GENOSTAFF, Cat No.STF-01), and embedded in paraffin by the proprietary procedures.

ISH was performed with the Ventana HX system (Ventana Medical Systems, Inc., Tucson, AZ, USA). Entire EST inserts were amplified by PCR using ExTaq (TaKaRa, Kyoto, Japan) in a $50 \mu$ reaction mixture using M13 forward and reverse primers. Amplification was performed as follows: $3 \mathrm{~min}$ at $94{ }^{\circ} \mathrm{C}$ for initial denaturation, 35 cycles of $94{ }^{\circ} \mathrm{C}$ denaturing for $30 \mathrm{~s}$ $60{ }^{\circ} \mathrm{C}$ annealing for $30 \mathrm{~s}$, and $72^{\circ} \mathrm{C}$ extension for $1 \mathrm{~min}$, followed by a final extension at $72{ }^{\circ} \mathrm{C}$ for $10 \mathrm{~min}$. Quality and quantity of the purified PCR product was confirmed by $1 \cdot 2 \%$ agarose gel electrophoresis. Antisense and sense RNA probes were labeled using the T7/T3 digoxigenin RNA labeling kit (Roche Diagnostics, Indianapolis, IN, USA), according to the manufacturer's instructions. Sections were pre-treated and hybridized with a Ventana RiboMap kit (Ventana Medical Systems) on the automated Ventana HX system Discovery. Detection of hybrids was performed with a digoxigenin nucleic acid detection kit (Boehringer Mannheim, Germany) following the manufacturer's instructions. Sections were then dehydrated through an ethanol series (80, 90 and 100\% ethanol, for 1 min each) and washed for $1 \mathrm{~min}$ in xylene before mounting in malinol mounting medium (Muto Pure Chemicals Ltd, Tokyo, Japan).

\section{Results and discussion}

The expression profile of genes in pancreatic islets of experimental animals will greatly complement human studies of functional genomics of the tissue and the genetics of its disease states. This study both establishes a first molecular inventory of rat pancreatic islets and reveals a number of novel genes, the expression of which has not previously been described. These should provide important insights into the entire transcriptome of endocrine pancreas and be an immensely valuable aid to the improvement of genomic annotation.

\section{Large-scale collection of ESTs from rat pancreatic islet and RINm5F cells}

A total of 40710 clones randomly selected from the two cDNA libraries were partially sequenced from the 3 -end. Sequences containing less than $1 \%$ ambiguous bases longer than $200 \mathrm{bp}$ were subjected to BLASTN database search. Contaminated genomic sequences, e.g. repetitive sequences, (1967 clones) and mitochondrial DNAs (4633 clones), were removed from the pool of sequences, resulting in a collection of 34110 ESTs comprising 22310 known and 11800 unknown sequences. Our previous study of 1000 ESTs from human pancreatic islets, the purity of which was 
Table 1 Redundancy of 3 '-ESTs from rat pancreatic islet and RINm5F cells

\begin{tabular}{|c|c|c|c|}
\hline & Islet & RINm5F & Islet \& RINm5F \\
\hline \multicolumn{4}{|c|}{ Redundancy } \\
\hline$>1000$ & 1 & 0 & 1 \\
\hline 101-1000 & 1 & 3 & 7 \\
\hline $51-100$ & 3 & 6 & 15 \\
\hline $31-50$ & 6 & 12 & 28 \\
\hline $21-30$ & 21 & 31 & 69 \\
\hline $11-20$ & 87 & 138 & 253 \\
\hline $6-10$ & 228 & 299 & 533 \\
\hline $3-5$ & 1039 & 1113 & 1878 \\
\hline 2 & 1556 & 1593 & 2075 \\
\hline 1 & 3088 & 3065 & 5547 \\
\hline
\end{tabular}

The 40710 ESTs were generated by sequencing cDNA clones from the $3^{\prime}$-ends. After clustering analysis, a total of 6030 and 6260 independent groups were obtained from pancreatic islet and RINm5F cells, respectively.

estimated to be $\sim 90 \%$ by microscopic examination and protein analysis, identified 13, 12, 6, and 9 ESTs for major exocrine genes for $\alpha$-amylase, elastase, pancreatic lipase, and trypsinogen respectively (Takeda et al. 1993). Since only 2, 8, 2, and 5 clones for these exocrine genes respectively, were found in the $\sim 20000$ EST sequences of this study, the possibility of contamination from exocrine cells appears to be quite negligible $(\sim 0 \cdot 1 \%)$. This estimation of purity is consistent with that of the protein analysis described above. Because large-scale sequencing based on random isolation of clones generates high redundancy, clustering analysis was performed to assemble the sequences into nonredundant sequence groups. A total of 6030 and 6260 independent groups were obtained from pancreatic islet and RINm5F cells respectively, and the pattern of redundancy was similar between the two sources (Table 1 \& 2). Together, 10406 non-redundant sequences comprising 4859 clusters of sequences and 5547 singletons were obtained representing 4078 known genes and 6328 unknown genes. Since only 1896 distinct genes $(18 \%)$ were found to overlap in the normal islets and RINm5F cells, this large-scale sequencing using two distinct cDNA sources was quite effective in identifying a larger number of non-redundant sequences. Studies of

Table 2 Summary of non-redundant $3^{\prime}$-ESTs and/or 5'-ESTs

\begin{tabular}{|c|c|c|c|}
\hline & Known & Unknown & Total \\
\hline Islet only & 1165 & 2981 & 4146 \\
\hline RINm5F only & 1569 & 2807 & 4376 \\
\hline Islet \& RINm5F & 1344 & 540 & 1884 \\
\hline Total & 4078 & 6328 & 10406 \\
\hline
\end{tabular}

The 6030 and 6260 non-redundant sequences from pancreatic islet and RINm5F cells, respectively, were subjected to BLASTN database search at the National Center for Biotechnology Information. the number of different mRNA sequences in a cell suggest that a typical higher eukaryotic cell synthesizes 10000 to 20000 different proteins (Alberts et al. 1994), so this approach covered at least $50 \%$ of the possible protein-coding genes. As in similar large-scale cDNA sequencing studies carried out in other tissues, about $50 \%$ of the clones obtained are derived from genes not functionally annotated. These unknown clones were re-sequenced from the $5^{\prime}$-end, and the 5512 clones sequenced successfully were also subjected to database search. As a result, 1404 sequences representing 502 distinct transcripts showed perfect identity to known genes, so the 3 '-end sequences of these clones clearly are not contained in the cDNA sequences deposited in the nucleotide databases. These ESTs were then assigned to the known group. All representative EST sequences obtained from each cluster were deposited in the public database to be freely available to all researchers (DDBJ accession No. BP464981-BP504629).

\section{Characterization of known genes in pancreatic islet and RINm5F cells}

The ESTs showing identity or high similarity to known genes were classified into seven major categories on the basis of putative general functions of the protein encoded, as described previously (categories: cell division, cell signaling/communication, cell structure/ motility, cell/organism defense, gene/protein expression, metabolism, and unclassified). In total, 3951 out of 4078 known genes were represented in the classified data set (online supplement). The largest category of genes was gene/protein expression (26.4\%). Successively smaller categories were cell signaling and communication $(19 \cdot 0 \%)$, metabolism $(16 \cdot 8 \%)$, cell structure/ motility $(7 \cdot 7 \%)$, cell/organism defense $(7 \cdot 5 \%)$, and cell division $(5 \cdot 6 \%)$. ESTs lacking sufficient information to be classified constituted the remainder, unclassified $(16 \cdot 9 \%)$. To further analyze the molecular complexity, each major category was subdivided according to the putative specific functions of the proteins (Table 3, also see online supplement). For example, the largest category, gene/protein expression, was subdivided into eight subgroups. Of these, transcription factor constituted the largest number of non-redundant genes $(416$ genes by $1209 \mathrm{ESTs})$. The transcription factors include PDX-1, BETA2/NeuroD, HNF-4 $\alpha$, Nkx-2·2, Nkx-6·1, and Isl-1 etc, all of which are important for pancreatic development and islet-specific functions, and the first three of which are the causal genes for monogenic forms of diabetes, MODY4, MODY6, and MODY1 (Fajans et al. 2001). The other genes for transcription factors also are plausible candidates for diabetogenes or genes responsible for $\beta$-cell specific functions.

In this study, $60.8 \%$ of the non-redundant $\mathrm{EST}_{\mathrm{S}}$ did not match any of the known genes in the nucleotide 
Table 3 Functional categories of proteins encoded by non-redundant ESTs

\begin{tabular}{|c|c|c|c|c|c|}
\hline & Subcategory & Islet only & RINm5F only & Islet \& RINm5F & Tota \\
\hline \multicolumn{6}{|l|}{ Functional category } \\
\hline \multirow[t]{6}{*}{ Cell division } & General & 18 & 13 & 21 & 52 \\
\hline & DNA synthesis & 1 & 18 & 5 & 24 \\
\hline & Apoptosis & 13 & 15 & 18 & 46 \\
\hline & Cell cycle & 13 & 36 & 19 & 68 \\
\hline & Chromosomal structure & 8 & 15 & 9 & 32 \\
\hline & Subtotal & 53 & 97 & 72 & 222 \\
\hline \multirow[t]{9}{*}{ Cell signaling } & Cell adhesion & 20 & 18 & 12 & 50 \\
\hline & Channel & 15 & 21 & 19 & 55 \\
\hline & Effectors & 23 & 24 & 24 & 71 \\
\hline & Hormone & 31 & 28 & 25 & 84 \\
\hline & Intracellular transducers & 50 & 53 & 62 & 165 \\
\hline & Metabolism & 2 & 1 & 3 & 6 \\
\hline & Protein modification & 56 & 43 & 55 & 154 \\
\hline & Receptor & 60 & 57 & 51 & 168 \\
\hline & Subtotal & 257 & 245 & 251 & 753 \\
\hline \multirow[t]{7}{*}{ Cell structure } & General & 9 & 17 & 16 & 42 \\
\hline & Contractile protein & 9 & 8 & 7 & 24 \\
\hline & Cytoskeletal & 23 & 26 & 39 & 88 \\
\hline & Extracellular matrix & 24 & 10 & 8 & 42 \\
\hline & Microtubule-associated & 17 & 23 & 17 & 57 \\
\hline & Vesicular transport & 15 & 19 & 19 & 53 \\
\hline & Subtotal & 97 & 103 & 106 & 306 \\
\hline \multirow[t]{6}{*}{ Cell defense } & Homeostasis (general) & 21 & 16 & 20 & 57 \\
\hline & DNA repair & 11 & 17 & 11 & 39 \\
\hline & Carrier protein & 22 & 24 & 22 & 68 \\
\hline & Stress response & 9 & 12 & 14 & 35 \\
\hline & Immunology & 47 & 23 & 27 & 97 \\
\hline & Subtotal & 110 & 92 & 94 & 296 \\
\hline \multirow[t]{9}{*}{ Gene expression } & RNA polymerase & 6 & 6 & 12 & 24 \\
\hline & RNA processing & 23 & 70 & 62 & 155 \\
\hline & Transcription factor & 136 & 173 & 107 & 416 \\
\hline & Targeting & 37 & 49 & 65 & 151 \\
\hline & Protein turnover & 34 & 28 & 47 & 109 \\
\hline & Ribosomal proteins & 17 & 20 & 87 & 124 \\
\hline & tRNA synthesis & 1 & 9 & 8 & 18 \\
\hline & Translation factor & 9 & 12 & 29 & 50 \\
\hline & Subtotal & 263 & 367 & 417 & 1047 \\
\hline \multirow[t]{10}{*}{ Metabolism } & General & 4 & 4 & 5 & 13 \\
\hline & Amino acid & 12 & 15 & 18 & 45 \\
\hline & Cofactors & 1 & 3 & 1 & 5 \\
\hline & Energy & 31 & 37 & 56 & 124 \\
\hline & Lipid & 39 & 51 & 49 & 139 \\
\hline & Nucleotide & 18 & 30 & 13 & 61 \\
\hline & Protein modification & 7 & 25 & 20 & 52 \\
\hline & Sugar & 19 & 81 & 30 & 130 \\
\hline & Transport & 25 & 36 & 31 & 92 \\
\hline & Subtotal & 156 & 282 & 223 & 661 \\
\hline \multicolumn{2}{|l|}{ Unclassified } & 197 & 300 & 169 & 666 \\
\hline \multicolumn{2}{|c|}{ Total No. of unique genes } & 1133 & 1486 & 1332 & 3951 \\
\hline
\end{tabular}

The ESTs showing identity or high similarity to known genes were classified into seven major categories on the basis of putative general functions of the protein encoded and each major category was further subdivided according to the putative specific functions of the proteins.

database. To identify novel rat genes encoding proteins structurally related to the known proteins, we performed BLASTX similarity search in the peptide databases using 5512 distinct ESTs. Of these, 127 represent rat homologs of genes identified in other species or new members of structurally related families in rat, the cut off for significant similarity being $P$ value of $10^{7}$ and similarity of $50 \%$ (Table 4). Functional analyses of the proteins encoded by these ESTs should clarify their novel roles in pancreatic islets. 
Table 4 Rat homologs of known genes and new members of gene families

\section{Gene}

Clone ID

RBC00545

RBC00858

RBC01084

RBC01744

RBC03066

RBC03516

RBC04593

RBC05170

RBC05610

RBC06005

RBC06162

RBC06680

RBC07890

RBC08283

RBC08942

RBC09487

RBC09605

RBC10109

RBC11646

RBC12591

RBC12830

RBC12840

RBC13044

RBC13272

RBC13530

RBC14441

RBC14577

RBC15029

RBC15417

RBC15535

RBC15661

RBC15731

RBC17834

RBC18842

RBC19899

RBC20402

RIN00207

RIN00228

RIN00249

RIN00488

RIN00804

RIN01128

RIN01130

RIN01171

RIN01386

RIN01389

RIN01548

RIN01784

RIN01794

RIN01840

RIN01932

RIN02139

RIN02177

RIN02445

RIN02483 3

IS904 breast carcinoma amplified sequence 3 homolog

retrovirus-related POL polyprotein

eukaryotic translation initiation factor 4 gamma

protein C20orf149 homolog

DNA transformation protein comF

hypothetical protein in acoE 3 'region

speckle-type POZ protein-like 1; POZ 56

protein

hypothetical protein 3

$14 \mathrm{kDa}$ phosphohistidine phosphatase

transposase for insertion sequence element

aurora-A kinase interacting protein

epsin 1

probable Pol polyprotein

G protein-coupled receptor 150

Egl nine homolog 2

hypothetical protein PP2447 homolog

putative NF-kappa-B activating protein

polyposis locus protein 1

immunoglobulin light chain variable region

chromosome 10 open reading frame 45

pORF2

methyl-accepting chemotaxis protein

ribonuclease $\mathrm{P}$ protein subunit p29

hypothetical protein KIAA0174

histone deacetylase 4 (HD4)

lactoylglutathione lyase

lymphocyte antigen Ly-6D precursor

protein C21orf5

POL polyprotein

amino-acid $\mathrm{ABC}$ transporter ATP-binding protein zinedin

collagen alpha $1(X)$ chain

probable transcriptional regulatory protein ygiX

nectin 4

probable 3-hydroxybutyryl-CoA dehydrogenase

hypothetical protein CGI-143

S-adenosylmethionine-dependent

methyltransferase

heat inducible transcriptional repressor protein

cell cycle control protein cwf15

hypothetical protein C5D6.06c

zinc finger protein 35 (Zfp-35)

tyrosine-protein kinase FLK

trigger factor (TF)

WD-repeat protein CGI-48

probable cation-transporting ATPase 1

L-lactate dehydrogenase

THAP domain protein 11

hypothetical protein KIAA0233

cell division protein $\mathrm{ftsH}$ homolog

hypothetical protein MG061

condensin complex subunit 2 (p105)

protein FAM3A precursor

ABC transporter ATP-binding protein

translation initiation factor IF3

splicing factor, arginine/serine-rich 2
Species

Homo sapiens

Mus musculus

Homo sapiens

Rattus norvegicus

Pseudomonas stutzeri

Rhodobacter sphaeroides

Rattus norvegicus

Rattus norvegicus

Rattus norvegicus

Pseudomonas putida

Rattus norvegicus

Rattus norvegicus

Rattus norvegicus

Mus musculus

Mus musculus

Mus musculus

Rattus norvegicus

Rattus norvegicus

Mus musculus

Homo sapiens

Mus musculus

Pseudomonas syringae

Homo sapiens

Rattus norvegicus

Rattus norvegicus

Rattus norvegicus

Rattus norvegicus

Homo sapiens

Trichosurus vulpecula

Thermobifida fusca

Rattus norvegicus

Mus musculus

synthetic construct

Mus musculus

Brucella melitensis

Rattus norvegicus

Mycoplasma mobile

Mycoplasma mobile

Mus musculus

Mus musculus

Rattus norvegicus

Rattus norvegicus

Mycoplasma pulmonis

Rattus norvegicus

Rattus norvegicus

Mesoplasma florum L1

Rattus norvegicus

Rattus norvegicus

Mycoplasma pulmonis

Mycoplasma pulmonis

Mus musculus

Mus musculus

Mycoplasma gallisepticum

Mycoplasma fermentans

Mus musculus

\begin{tabular}{|c|c|c|c|}
\hline \%SIM & $P$ value & Islet & RINm5F \\
\hline 0.72 & $2 \mathrm{E}-21$ & 1 & 1 \\
\hline 0.74 & $4 \mathrm{E}-42$ & 1 & 0 \\
\hline 0.66 & $5 \mathrm{E}-44$ & 1 & 0 \\
\hline 0.74 & $1 E-51$ & 1 & 0 \\
\hline 0.74 & $3 E-63$ & 1 & 0 \\
\hline 0.85 & $7 \mathrm{E}-48$ & 1 & 0 \\
\hline 0.72 & $3 E-32$ & 1 & 0 \\
\hline 0.78 & 7E-35 & 1 & 1 \\
\hline 0.64 & $9 \mathrm{E}-28$ & 3 & 0 \\
\hline 0.83 & $2 E-49$ & 1 & 0 \\
\hline 0.79 & $2 E-45$ & 2 & 0 \\
\hline 0.75 & $8 \mathrm{E}-38$ & 1 & 0 \\
\hline 0.71 & $5 E-43$ & 1 & 0 \\
\hline 0.81 & 4E-89 & 1 & 0 \\
\hline 0.75 & $2 E-07$ & 2 & 0 \\
\hline 0.75 & $4 \mathrm{E}-19$ & 1 & 1 \\
\hline 0.64 & $1 \mathrm{E}-43$ & 1 & 0 \\
\hline 0.79 & $2 \mathrm{E}-34$ & 1 & 0 \\
\hline 0.58 & $2 E-27$ & 1 & 0 \\
\hline 0.62 & $4 \mathrm{E}-41$ & 2 & 0 \\
\hline 0.64 & 1E-34 & 1 & 0 \\
\hline 0.64 & 9E-34 & 1 & 0 \\
\hline 0.64 & $2 E-19$ & 2 & 0 \\
\hline 0.76 & $5 \mathrm{E}-13$ & 3 & 0 \\
\hline 0.73 & $2 E-18$ & 1 & 0 \\
\hline 0.71 & $7 E-49$ & 2 & 0 \\
\hline 0.79 & $4 \mathrm{E}-38$ & 2 & 0 \\
\hline 0.85 & 7E-33 & 1 & 0 \\
\hline 0.71 & $9 \mathrm{E}-25$ & 2 & 0 \\
\hline 0.83 & $3 E-80$ & 1 & 0 \\
\hline 0.76 & $1 \mathrm{E}-22$ & 1 & 1 \\
\hline 0.66 & $3 E-64$ & 4 & 0 \\
\hline 0.72 & $2 E-53$ & 1 & 0 \\
\hline 0.75 & $8 E-73$ & 1 & 0 \\
\hline 0.81 & 3E-99 & 3 & 0 \\
\hline 0.64 & $4 \mathrm{E}-28$ & 1 & 0 \\
\hline 0.68 & $3 E-54$ & 0 & 2 \\
\hline 0.59 & $3 E-32$ & 0 & 1 \\
\hline 0.56 & $4 \mathrm{E}-12$ & 0 & 1 \\
\hline 0.86 & 3E-85 & 0 & 1 \\
\hline 0.62 & $5 \mathrm{E}-22$ & 0 & 1 \\
\hline 0.77 & $1 \mathrm{E}-42$ & 0 & 1 \\
\hline 0.65 & $7 \mathrm{E}-11$ & 0 & 1 \\
\hline 0.6 & 9E-28 & 0 & 1 \\
\hline 0.79 & 3E-82 & 0 & 1 \\
\hline 0.58 & 2E-20 & 0 & 1 \\
\hline 0.51 & 9E-22 & 0 & 1 \\
\hline 0.86 & $5 E-23$ & 0 & 1 \\
\hline 0.64 & 4E-32 & 0 & 1 \\
\hline 0.55 & 1E-34 & 0 & 4 \\
\hline 0.88 & $3 \mathrm{E}-14$ & 0 & 1 \\
\hline 0.87 & 5E-36 & 0 & 1 \\
\hline 0.74 & 7E-60 & 0 & 3 \\
\hline 0.62 & $8 \mathrm{E}-12$ & 0 & 2 \\
\hline 0.66 & 2E-59 & 0 & 2 \\
\hline
\end{tabular}


Table 4 Continued

Gene

Clone ID

RIN02532

RIN02603

RIN03035

RIN03110

RIN03339

RIN03427

RIN04062

RIN04617

RIN05010

RIN05122

RIN05229

RIN05576

RIN05605

RIN05994

RIN06079

RIN06155

RIN06262

RIN06288

RIN06497

RIN06650

RIN07055

RIN07707

RIN07765

RIN07973

RIN08083

RIN08132

RIN08211

RIN08412

RIN09109

RIN09205

RIN09341

RIN09690

RIN10182

RIN10530

RIN10621

RIN10814

RIN11008

RIN11027

RIN11030

RIN11408

RIN11741

RIN11820

RIN11856

RIN11899

RIN12027

RIN12420

RIN12461

RIN12488

RIN12608

RIN12715

RIN12733

RIN12794

RIN12806
ABC transporter permease protein MG188 homolog

protein c20orf172 homolog

probable nicotinate-nucleotide

adenylyltransferase

phospholipase A2 inhibitor gamma subunit B

hypothetical lipoprotein MPN288

putative Pol polyprotein

hypothetical protein MG148

probable cation-transporting P-type ATPase

testis-specific protein PBS13

hypothetical protein KIAA0036

E3 ubiquitin-protein ligase Nedd-4

TLM protein

RRS1 ribosome biogenesis regulator homolog

eukaryotic translation initiation factor $4 \mathrm{E}$

transporter

brain protein 14

slingshot 3

mitochondrial respiratory chain complexes

assembly protein

SCO2 protein homolog, mitochondrial precursor methionine aminopeptidase

retrovirus-related protease

TGF-beta induced apoptosis protein 2

methyl-CpG binding domain protein 6

splicing factor 3 subunit 1

$\mathrm{NADH}$-ubiquinone oxidoreductase chain $4 \mathrm{~L}$ signal recognition particle protein

forkhead box protein $\mathrm{K} 1$

meningioma-expressed antigen 6/11

hypothetical $45.0 \mathrm{kDa}$ protein in NOT1-MATAL2 region

TED protein

SRR1-like protein

30 S ribosomal protein S6

valyl-tRNA synthetase

protein transport protein SEC61 gamma subunit hypothetical protein KIAA0893

splicing factor, arginine/serine-rich 8

$\mathrm{G}$ protein-coupled receptor family $\mathrm{C}$ group 5 member $\mathrm{C}$

tRNA-splicing endonuclease subunit SEN54

protein yhgF

p53-associated parkin-like cytoplasmic protein protein disulfide isomerase precursor

polyhomeotic-like protein 1

translation initiation factor IF-1

fructose-bisphosphate aldolase

nuclear protein Hcc-1

60 S ribosomal protein L22

putative ATP-dependent RNA helicase T26G10

synaptic vesicle membrane protein VAT-1

homolog

glutaminyl-peptide cyclotransferase

probable RNA-dependent helicase p72

oligoendopeptidase $\mathrm{F}$ homolog

PSL10 protein

hypothetical protein ORF-1137

protein HI1455
Species

Mycoplasma gallisepticum

Mus musculus

Mycoplasma mobile

Rattus norvegicus

Mycoplasma gallisepticum

Rattus norvegicus

Ureaplasma parvum

Ureaplasma parvum

Rattus norvegicus

Rattus norvegicus

Cricetulus griseus

Mus musculus

Mus musculus

Rattus norvegicus

Mus musculus

Rattus norvegicus

Mycoplasma pulmonis

Mus musculus

Mycoplasma pulmonis

Homo sapiens

Rattus norvegicus

Rattus norvegicus

Mus musculus

Rattus norvegicus

Mycoplasma mobile

Homo sapiens

Rattus norvegicus

Rattus norvegicus

Mus musculus

Homo sapiens

Mycoplasma pulmonis

Mycoplasma pulmonis

Rattus norvegicus

Mus musculus

Mus musculus

Rattus norvegicus

Rattus norvegicus

Homo sapiens

Rattus norvegicus

Rattus norvegicus

Rattus norvegicus

Leptospira interrogans

Mycoplasma mobile

Mus musculus

Mus musculus

Rattus norvegicus

Rattus norvegicus

Rattus norvegicus

Rattus norvegicus

Mycoplasma pulmonis

Mus musculus

Rattus norvegicus

Mycoplasma pulmonis
$\%$ SIM

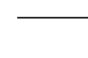

$P$ value

Islet

RINm5F

0.65

0.89

0.65

7E-11

2E-44

3E-26

0.84

0.56

0.77

0.69

0.55

0.78

0.86

0.86

0.64

0.67

0.7

0.61

0.79

0.73

2E-07

$4 \mathrm{E}-10$

2E-19

3E-22

$1 \mathrm{E}-31$

$5 \mathrm{E}-88$

$6 \mathrm{E}-76$

$4 \mathrm{E}-48$

$1 \mathrm{E}-26$

3E-35

$3 \mathrm{E}-13$

$8 \mathrm{E}-12$

$6 \mathrm{E}-47$

3E-37

$0.76 \quad 4 \mathrm{E}-45$

0.7

0.54

0.62

0.61

0.55

0.72

0.72

0.66

0.71

0.85

$1 \mathrm{E}-37$

2E-12

2E-51

$1 \mathrm{E}-24$

2E-35

$7 \mathrm{E}-23$

$1 \mathrm{E}-21$

$1 \mathrm{E}-26$

2E-27

3E-79

0.87

0.83

0.55

0.87

0.7

0.81

0.51

0.73

5E-85

2E-42

2E-17

$5 \mathrm{E}-40$

$5 \mathrm{E}-20$

9E-93

5E-15

$8 \mathrm{E}-43$

0.86

0.89

0.84

0.74

0.82

0.74

0.8

0.72

0.81

0.8

0.73

$6 \mathrm{E}-98$

9E-93

9E-44

7E-35

3E-54

9E-12

6E-52

2E-67

2E-39

4E-83

8E-65

0.78

0.64

0.68

0.77

0.72

0.74

7E-90

8E-62

$8 \mathrm{E}-58$

7E-80

2E-32

7E-50 
Table 4 Continued

\section{Gene}

Clone ID

RIN12897

RIN12933

RIN13293

RIN13306

RIN13714

RIN13766

RIN13865

RIN14137

RIN14394

RIN14494

RIN14758

RIN15622

RIN16005

RIN16184

RIN16330

RIN16398

RIN16446

RIN16588

RIN16592

Csr1 dimethyladenosine transferase

zinc finger protein 23

serine hydroxymethyltransferase

peripherin

seryl-tRNA synthetase

UBA/UBX $33.3 \mathrm{kDa}$ protein

splicing factor 1

condensin subunit 1

hypothetical protein KIAA0117

general transcription factor $3 \mathrm{C}$ polypeptide 5

Crumbs protein homolog 1

hypothetical protein C19A8.09

single-strand binding protein 1

zinc finger protein 510

$60 S$ ribosomal protein $\mathrm{L} 7$

single-strand binding protein

putative adenosylhomocysteinase 2

thymidine phosphorylase
Species

Mycoplasma gallisepticum

Mus musculus

Rattus norvegicus

Mus musculus

Mycoplasma pulmonis

Rattus norvegicus

Mus musculus

Mus musculus

Cricetulus griseus

Rattus norvegicus

Rattus norvegicus

Mus musculus

Rattus norvegicus

Rattus norvegicus

Mus musculus

Rattus norvegicus

Rattus norvegicus

Homo sapiens

Mycoplasma pulmonis
$\%$ SI

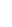

$P$ value Islet RINm5F

0.67

0.79

0.65

0.7

0.82

0.65

0.73

0.62

0.82

0.77

0.73

0.6

0.89

0.67

0.83

0.83

0.87

0.89

0.66

\section{$5 \mathrm{E}-36$}

$4 \mathrm{E}-63$

$4 \mathrm{E}-93$

$1 \mathrm{E}-42$

3E-62

$1 \mathrm{E}-54$

$6 \mathrm{E}-23$

2E-33

$8 \mathrm{E}-12$

7E-81

$1 \mathrm{E}-48$

7E-08

4E-11

$6 \mathrm{E}-13$

2E-19

1E-32

3E-13

2E-07

3E-20
02

12

$0 \quad 1$

$0 \quad 1$

$0 \quad 1$

$0 \quad 1$

02

$0 \quad 1$

$0 \quad 1$

0

11

$0 \quad 1$

0 2

11

02

13

0

02

The unknown clones after BLASTN search in the nucleotide databases were re-sequenced from the $5^{\prime}$-end and then subjected to BLASTX search in the peptide databases. The cut off used for significant similarity was $P$ value of $10^{-7}$ and similarity of $50 \%$. The clone IDs RBC and RIN show ESTs from pancreatic islet and RINm5F cells, respectively.

To identify genes that had not been determined to be expressed, the sequences showing no significant match to any of the annotated genes were further compared with dbEST entries from other tissues, revealing 896 expressed genes that have not appeared in the database. These 896 putatively novel genes were analyzed in the context of recently determined rat genomic sequences (Rat Genome Sequencing Project Consortium 2004). Of these genes, 779 were encoded by exon-intron organization in the corresponding genomic sequences. However, since the transcripts of many of these genes were barely detectable by in situ hybridization, they may be expressed at low levels, at least in adult islets. Because chemiluminescence-based in situ detection of mRNAs is not sufficiently sensitive, a large-scale RT-PCR analysis is presently in progress in our laboratory to elucidate the tissue distribution. The expression of 71 of the other 117 sequences was uncertain due to their ambiguous genomic structure. The remainder could be derived from possible pseudogenes or retroposons, as the corresponding sequences in the genome are flanked by AT-rich sequences that were recognizable by oligo-dT priming in the process of cDNA synthesis.

\section{Characterization of differentially expressed genes}

The immunoreactive insulin (IRI) content of rat normal $\beta$-cells has been reported to be $\sim 8000 \mathrm{pmol} / 10^{6}$ cells, while the RINm5F cell line used in this study has been estimated to contain a very low level of IRI $\left(0.43 \mathrm{pmol} / 10^{6}\right.$ cells $)$ and a much lower number of secretory granules (Kayo et al. 1997). Accordingly, the expression levels of the genes involved in insulin synthesis and secretion in RINm5F cells should markedly differ from those of the normal $\beta$-cells. In addition, since the RINm5F cells were derived from radiation-induced tumor cells and exhibited a decrement of well-differentiation (Gazdar et al. 1980, Philippe et al. 1987, Kayo et al. 1997), the expression levels of the genes involved in cell differentiation and tumorigenesis may also be altered. The relative frequencies of ESTs have been shown to reflect the average level of expression of the corresponding mRNAs in the tissues examined (Lee et al. 1995). As pancreatic islet cells are mostly $\beta$-cells, the expression profile of insulin-related genes in the two cDNA sources (of same size) can be compared to identify differentially expressed genes. The EST frequencies for most of the house-keeping genes were similar in the two cDNA libraries, suggesting that such comparison of EST frequencies is reasonable. Over 2-fold differences in frequency between the two libraries were found in 204 genes (higher EST>10 times). The direction of change in mRNA levels in these ESTs observed by comparison of the EST frequency and the TaqMan semi-quantitative analysis was quite parallel, while the magnitude of the change was not correlated. The representative results for some of the ESTs ( $>15$ times) are shown in Fig. 1. Previously, similar comparative analysis using $\sim 6000$ ESTs from two different conditions of PG-12 cells was performed (Lee et al. 1995). The study found the ratio of EST frequencies between the two cDNA sources to be 


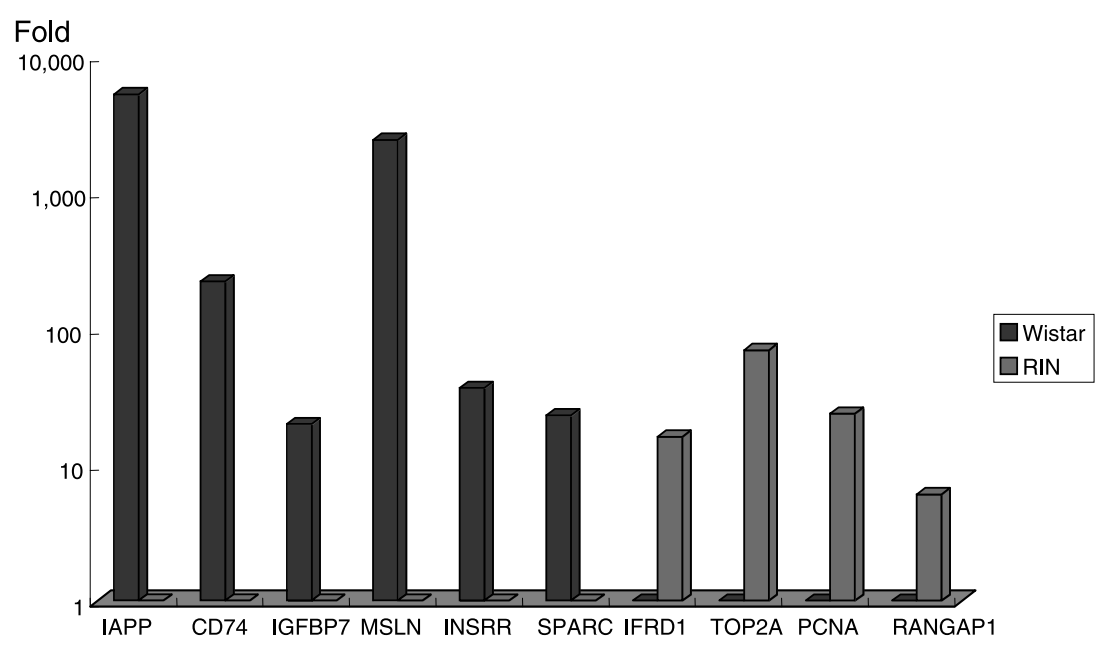

Figure 1 Expression profiles of the representative genes abundant either in islet (from Wistar rats) or in RINm5F cells. The expression levels of the genes were estimated by real-time quantitative RT-PCR using the TaqMan system. The mRNA for major $\alpha$-globin could not be efficiently detected by the TaqMan system in its standard range for unknown reasons.

correlated with the Northern blot analysis, except for the low-frequency ESTs. Thus, the genes of interest that are expressed at least at moderate levels also should be examined by semi-quantitative analysis before further analysis.

We focus on the seven genes exhibiting high expression (>15 times) in islet but no expression in RINm5F cells (Table 5), since they may well play a specific role in insulin synthesis and secretion. Expression of all of the genes examined was confirmed in pancreatic $\beta$ cells by in situ hybridization, although the expression was not restricted to $\beta$ cells (Fig. 2A). The expression of the high EST clones ( $>15$ times) in RINm5F cells and none in islets was found only outside the islets by in situ hybridization (Fig. 2B). These patterns of expression are consistent with a previous assumption that over $90 \%$ of the genes are house-keeping and are expressed at various levels in many tissues. Receptors of the insulin/insulin-like growth factor (IGF) family have been implicated both in the regulation of pancreatic $\beta$-cell growth and insulin secretion. IRR, an orphan receptor of the insulin receptor subfamily, is expressed at a considerably higher level in pancreatic $\beta$ cells (Hirayama et al. 1999), and a decrease in the mRNA level was found in diabetic GK rats (N Shihara, Y Horikawa, J Takeda, unpublished observations). However, since glucose-stimulated insulin secretion and embryonic $\beta$ cell development have been shown to occur normally in mice lacking IRR (Kitamura et al. 2001), decreased expression of IRR alone may be insufficient for the development of diabetes. To understand the functional properties of IRR in pancreatic $\beta$ cells, it is important to identify its possible ligand and functional partners. Mesothelin, produced by mesothelial cells, has been suggested to play a role in cellular adhesion in ovarian cancer cells (Scholler et al. 1999). Since this EST was not found in cultured RINm5F cells, methothelin may well be unimportant in single-cell growth without cell adhesion and not directly involved in endocrine

Table 5 Genes abundantly identified either in islet or in RINm5F cells

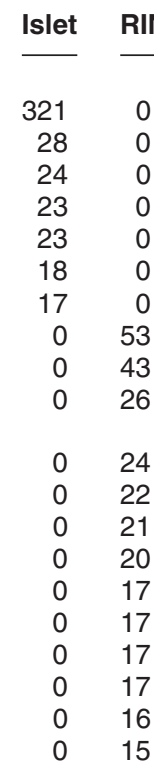

Genes with high (>15 times) expression in either islet and RINm5F and none of the other, are listed.

Journal of Molecular Endocrinology (2005) 35, 1-12

\section{Gene}

Islet amyloid polypeptide (amylin)

CD74

Follistatin-like protein (mac25)

Mesothelin

Major alpha-globin

Insulin receptor-related receptor (IRR)

Osteonectin

Interferon-related developmental regulator 1

DNA topoisomerase II alpha

Proliferating cell nuclear antigen

Glycosyl-phosphatidyl-inositol-anchored

protein homolog

Casein kinase 1 gamma 2 isoform

Ran-GTPase activating protein 1

Heat shock $70 \mathrm{kD}$ protein 5

High mobility group protein 17

Phosphoglycerate mutase type B subunit

Synaptic regulatory protein RIM2beta

Heat shock protein 60

HLA-B associated transcript 2

Secretory carrier membrane protein 3 


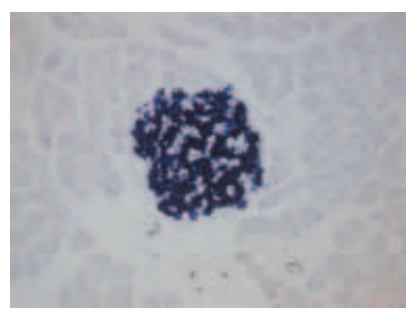

INS

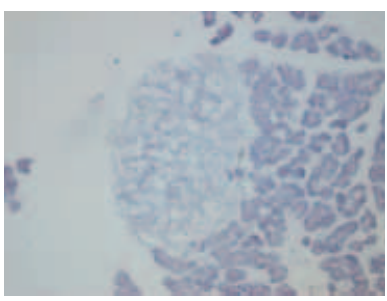

MSLN

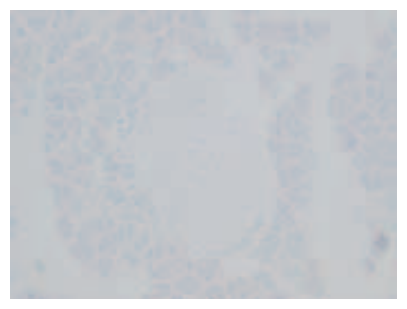

TOP2A

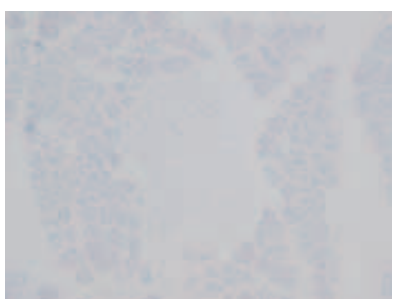

RANGAP1

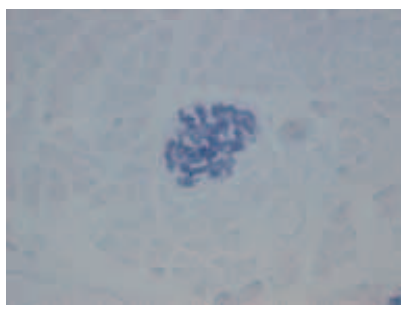

IAPP

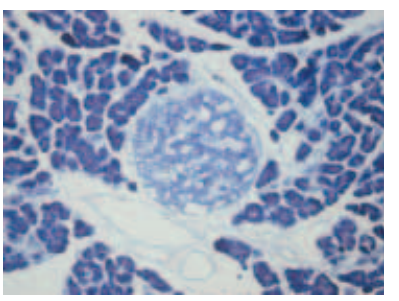

HBA1

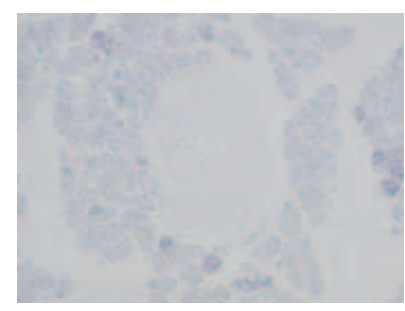

PCNA

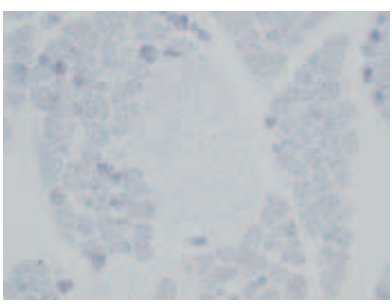

HSPA5

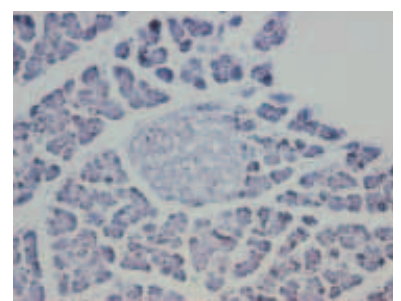

CD74

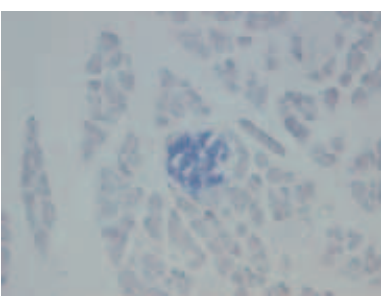

INSRR

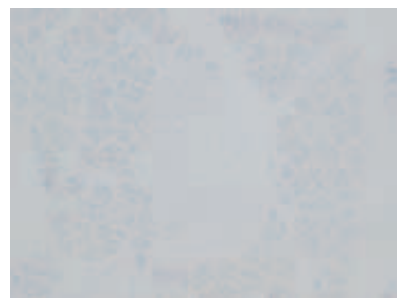

GPIAP1

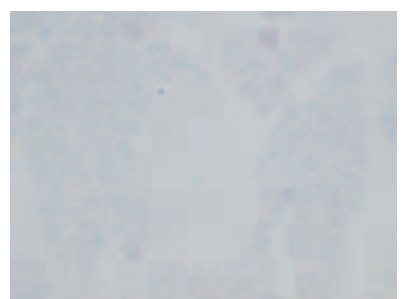

HMGN2

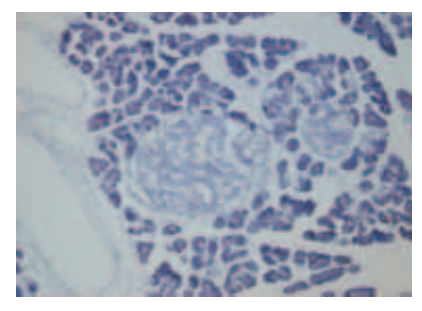

IGFBP7

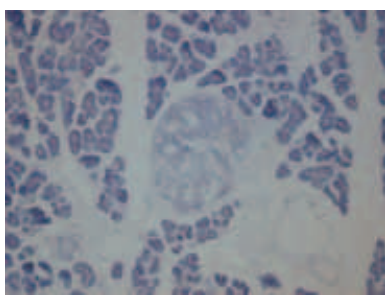

SPARC

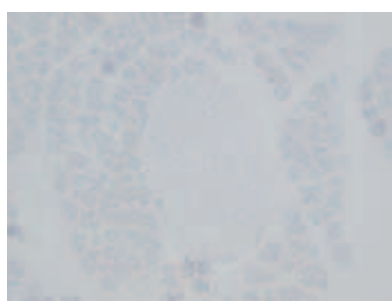

CSNK1G2

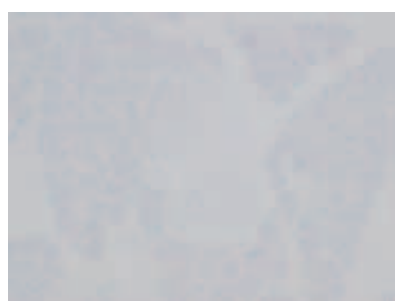

PGAM1

Figure 2 (A) In situ hybridization of genes with high expression in islet and none in RINm5F cells (A). The representative results of seven highly expressed genes (>15 times) and the insulin gene are shown. (B) In situ hybridization of genes with high expression in RINm5F cells and none in islet. 
tumorigenesis. CD74, which is transitorily associated with class II histocompatibility antigens during intracellular transport (Claesson et al. 1983), also was found to be highly expressed in human islet tumor in our previous study (Jin et al. 2003). Since this islet tumor exhibited features of moderately differentiated islet cells, despite markedly reduced insulin secretion, in contrast to RINm5F cells, CD74 might be related to the degree of cell differentiation. Osteonectin is reported to be abundantly expressed in adipose tissue. Although the molecule has not been reported to be expressed in islets, in situ hybridization showed that its expression is moderate in islet cells and diffuse throughout the pancreas of normal Wistar rat. It has been reported that absence of osteonectin leads to an increase in the size of individual adipocytes as well as in the number of adipocytes per fat pad (Bradshaw et al. 2003). Thus, osteonectin might be related to $\beta$ cell mass and growth rather than insulin synthesis and secretion. As these genes might contribute to the development of islet cell specificity, their functional significance should be examined in various conditions of pancreatic islets.

\section{Applications of rat ESTs for islet studies}

In this study, we describe a collection of 40710 rat pancreatic islet-related ESTs representing 10406 different transcripts. This is the first report describing a systematic collection of rat expressed genes from pancreatic islets and a $\beta$-cell line. Since DNA microarray technology relies largely on the rapid growth of the EST databases, these newly identified expressed genes should facilitate analysis of differential gene expression in pancreatic islets under various conditions. At present, only the PanChip microarray, which was prepared using 3400 cDNA sequences from mouse whole pancreas, is available as a tissue-specific microarray for islet studies (Scearce et al. 2002). Accordingly, the establishment of islet-specific DNA microarrays for the rat should be especially important in the analysis of the transcriptome of diabetic rats such as GK and OLETF, and is presently underway in our laboratory. Another advantage of the large-scale collection of EST clones is that the cDNA fragments obtained can be used as hybridizing probes for Northern blotting or in situ hybridization to analyze the size and number of alternatively spliced transcripts and their local tissue distribution. However, it is possible that a small fraction of the ESTs obtained might be contaminated from other cell types such as endothelial cells or blood. Indeed, our preliminary trial of non-isotopic in situ hybridization using rat ESTs was found to be quite effective for analysis of mRNA expression in pancreatic islets. A large-scale in situ hybridization of rat islet mRNAs is also presently in progress in our laboratory. Functional analysis of a wide spectrum of islet-specific genes and genes highly abundant or less abundant in islets identified by this approach might clarify the molecular mechanisms underlying the differentiation of islet cells, tumorigenesis, and the pathogenesis of diabetes, as well as lead to new therapies for the improvement and regeneration of $\beta$-cell function through manipulation of gene expression and gene products.

In addition, as the genome sequence analysis of the Brown Norway rat recently has been completed (Rat Genome Sequencing Project Consortium 2004), the results of this study should be helpful in annotating the genes actually expressed in the rat genome and thus provide further insight into mammalian evolution of genes involved in tissue-specificity of endocrine pancreas.

\section{Acknowledgements}

We thank S Oike, R Kawakami, Y Yaginuma, I Uda, Y Ibe, and T Takahashi for excellent assistance. This study was supported by Grant-in-Aid for Scientific Research and for Scientific Research on Priority Areas (C) "Medical Genome Science" from the Japanese Ministry of Science, Education, Sports, Culture and Technology and by a Health and Labor Science Research Grant for Research on Human Genome and Tissue Engineering from the Japanese Ministry of Health, Labor and Welfare, the Naito Foundation and the Yamanouchi Foundation. The authors declare that there is no conflict of interest that would prejudice the impartiality of this scientific work.

\section{References}

Alberts B, Bray D, Lewis J, Raff M, Roberts K \& Watson JD 1994 The cell nucleus. In: Molecular Biology of the Cell. pp 335-400. New York: Garland.

Altschul SF, Madden TL, Schaffer AA, Zhang J, Zhang Z, Miller W \& Lipman DJ 1997 Gapped BLAST and PSI-BLAST: a new generation of protein database search programs. Nucleic Acids Research 25 3389-3402.

Bernal-Mizrachi E, Cras-Meneur C, Ohsugi M \& Permutt MA 2003 Gene expression profiling in islet biology and diabetes research. Diabetes Metabolism Research Review 19 32-42.

Bonner-Weir S \& Sharma A 2002 Pancreatic stem cells. Fournal of Pathology 197 519-526.

Bradshaw AD, Graves DC, Motamed K \& Sage EH 2003 SPARC-null mice exhibit increased adiposity without significant differences in overall body weight. PNAS 100 6045-6050.

Claesson L, Larhammar D, Rask L \& Peterson PA 1983 cDNA clone for the human invariant gamma chain of class II histocompatibility antigens and its implications for the protein structure. PNAS 80 7395-7399.

Dor Y, Brown J, Martinez OI \& Melton DA 2004 Adult pancreatic $\beta$-cells are formed by self-duplication rather than stem-cell differentiation. Nature 429 41-46. 
Edlund H 2002 Pancreatic organogenesis - Developmental mechanisms and implications for therapy. Nature Genetics Review $\mathbf{3}$ 524-532.

Fajans SS, Bell GI \& Polonsky KS 2001 Molecular mechanisms and clinical pathophysiology of maturity-onset diabetes of the young. New England Fournal of Medicine 345 971-980.

Gazdar AF, Chick WL, Oie HK, Sims HL, King DL, Weir GC \& Lauris V 1980 Continuous, clonal, insulin- and somatostatinsecreting cell lines established from a transplantable rat islet cell tumor. PNAS 77 3519-3523.

Hirayama I, Tamemoto H, Yokota H, Kubo SK, Wang J, Kuwano H, Nagamachi Y, Takeuchi T \& Izumi T 1999 Insulin receptor-related receptor is expressed in pancreatic beta-cells and stimulates tyrosine phosphorylation of insulin receptor substrate-1 and -2. Diabetes 48 1237-1244.

Hogenesch JB, Ching KA, Batalov S, Su AI, Walker JR, Zhou Y, Kay SA, Schultz PG \& Cooke MP 2001 A comparison of the Celera and Ensembl predicted gene sets reveals little overlap in novel genes. Cell 106 413-415.

International Human Genome Sequencing Consortium 2001 Initial sequencing and analysis of the human genome. Nature $\mathbf{4 0 9}$ 860-921.

Jin L, Wang H, Narita T, Kikuno R, Ohara O, Shihara N, Nishigori T, Horikawa Y \& Takeda J 2003 Expression profile of mRNAs from human pancreatic islet tumors. Fournal of Molecular Endocrinology 31 519-528.

Kayo T, Sawada Y, Suda M, Konda Y, Izumi T, Tanaka S, Shibata H \& Takeuchi T 1997 Proprotein-processing endoprotease furin controls growth of pancreatic $\beta$-cells. Diabetes 46 1296-1304.

Kitamura T, Kido Y, Nef S, Merenmies J, Parada LF \& Accili D 2001 Preserved pancreatic $\beta$-cell development and function in mice lacking the insulin receptor-related receptor. Molecular and Cellular Biology 21 5624-5630.

Lee NH, Weinstock KG, kirkness EF, Earle-Hughes JA, Fuldner RA, Marmaros S, Glodek A, Gocayne JD, Adams MD, Kerlavage AR, Fraser CM \& Venter JC 1995 Comparative expressed-sequence- tag analysis of differential gene expression profiles in PC-12 cells before and after nerve growth factor treatment. PNAS 92 8303-8307.

Ma H-T, Kato M \& Tatemoto K 1996 Effects of pancreastatin and somatostatin on secretagogues-induced rise in intracellular free calcium in single rat pancreatic islet cells. Regulatory Peptide $\mathbf{6 1}$ 143-148.

Mouse Genome Sequencing Consortium 2002 Initial sequencing and comparative analysis of the mouse genome. Nature $\mathbf{4 2 0}$ 520-562.

Philippe J, Chick WL \& Habener JF 1987 Multipotential phenotypic expression of genes encoding peptide hormones in rat insulinoma cell lines. Fournal of Clinical Investigation 79 351-358.

Rat Genome Sequencing Project Consortium 2004 Genome sequence of the Brown Norway rat yields insights into mammalian evolution. Nature 428 493-521.

Scearce LM, Brestelli JE, McWeeney SK, Lee CS, Mazzarelli J, Pinney DF, Pizarro A, Stoeckert CJ Jr, Clifton SW, Permutt MA, Brown J, Melton DA \& Kaestner KH 2002 Functional genomics of the endocrine pancreas. The pancreas clone set and PanChip, new resources for diabetes research. Diabetes 51 1997-2004

Scholler N, Fu N, Yang Y, Ye Z, Goodman GE, Hellstrom KE \& Hellstrom I 1999 Soluble member(s) of the mesothelin/ megakaryocyte potentiating factor family are detectable in sera from patients with ovarian carcinoma. PNAS 96 11531-11536.

Takeda J, Yano H, Eng S \& Bell GI 1993 A molecular inventory of human pancreatic islets: sequence analysis of $1000 \mathrm{cDNA}$ clones. Human Molecular Genetics 2 1793-1798.

Venter JC, Adams MD, Myers EW, Li PW, Mural RJ, Sutton GG, Smith HO, Yandell M, Evans CA, Holt RA, et al. 2001 The sequence of the human genome. Science 291 1304-1351.

Received 17 March 2005

Accepted 29 April 2005

Made available online as an Accepted Preprint 16 May 2005 